\title{
Electrocardiographic response to enzyme replacement therapy for Pompe disease
}

Annette K. Ansong, $M D^{1}$, Jennifer S. Li, $M D^{1}$, Eva Nozik-Grayck, $M D^{3}$, Richard Ing, MB, ChB ${ }^{4}$, Richard M. Kravitz, $M D^{5}$, Salim F. Idriss, $M D, P h D^{1}$, Ronald J. Kanter, $M D^{1}$, Henry Rice, $M D^{6}$, Y. T. Chen, MD, $P h D^{2}$, and Priya S. Kishnani, $M D^{2}$

\begin{abstract}
Purpose: Electrocardiogram (ECG) abnormalities are universal in infantile Pompe disease or glycogen storage disease type II, a fatal genetic muscle disorder caused by deficiency of acid $\alpha$-glucosidase (GAA). Hallmarks of this disease include a shortened PR interval, an increased QT dispersion (QTd), and large left ventricular (LV) voltages. We evaluated the effect of recombinant human GAA (rhGAA) enzyme replacement therapy (ERT) on these ECG parameters in patients with infantile-onset Pompe disease. Methods: A total of 134 ECGs were evaluated from 19 patients ( 5 females and 14 males) with a median age of 5.5 months at the time of enrollment in open-label clinical trials exploring the safety and efficacy of ERT at a single center from 1999 to 2004. rhGAA was purified from genetically engineered Chinese hamster ovary cells overproducing GAA and infused intravenously at doses ranging from $10 \mathrm{mg} / \mathrm{kg}$ per week to 20 to $40 \mathrm{mg} / \mathrm{kg}$ every 2 weeks in patients with infantile-onset Pompe disease. The PR interval, QTd (longest to shortest QT), and LV voltage (SV1+RV6) were blindly determined by two independent observers. Results: The median follow-up period was 6 months (range 2-30 months). The PR interval lengthened from $83(42-110) \mathrm{ms}$ to $107(95-130) \mathrm{ms}(P<.001)$, and the QTd decreased from $83(40-125) \mathrm{ms}$ to 53 $(20-80) \mathrm{ms}(P=.003)$. There were significant decreases in LV voltage $(67[17-83] \mathrm{mV}$ vs. 48 [18-77] $\mathrm{mV}, P=$ $.03)$, which correlated with decrease in LV mass on two-dimensional echocardiogram. There was no evident change in the QTc interval (429 [390-480] ms vs. 413 [370-450] ms, $P=$ not significant). Conclusion: rhGAA ERT for infantile Pompe disease results in an increase in PR interval and a decrease in both the QTd and the LV voltage. These results suggest that these ECG parameters may be useful markers of the severity of cardiac disease and the response to ERT treatment in patients with infantile Pompe disease. Genet Med 2006:8(5):297-301.
\end{abstract}

Key Words: Pompe disease, ERT, rhGAA, short PR interval, QT dispersion

Pompe disease or glycogen storage disease type II is an autosomal recessive disorder caused by a deficiency of acid $\alpha$-glucosidase (GAA) (acid maltase), a glycolytic lysosomal enzyme. This enzyme defect results in lysosomal glycogen accumulation in multiple tissues and cell types, with cardiac, skeletal, and smooth muscle cells being the most seriously affected. ${ }^{1}$ Complete deficiency results in infantile Pompe disease, which is uniformly fatal, with death usually by 1 year of age. In its most severe form, the infantile form of the disease is characterized by massive cardiomegaly, macroglossia, progressive skeletal muscle weakness, and resultant marked hypotonia. Glycogen accumulation in cardiac muscle causes a hypertrophic cardiomyopathy, which progresses to a dilated cardiomyopathy. Increasing left ventricular (LV) thickness can also lead to

\footnotetext{
From the ${ }^{1}$ Divisions of Pediatric Cardiology, ${ }^{2}$ Genetics, ${ }^{3}$ Critical Care, ${ }^{4}$ Anesthesiology, ${ }^{5}$ Pulmonary, and ${ }^{6}$ Surgery, Department of Pediatrics, Duke University Medical Center, Durham, North Carolina.

Received: June 10, 2005

Accepted: October 31, 2005.

Priya S. Kishnani, MD, Division of Medical Genetics, Duke University Medical Center, Bell Building Room 237, Trent Drive, Durham, NC.

DOI: 10.1097/01.gim.0000195896.04069.5f
}

obstruction of the LV outflow tract. At autopsy, the heart can be up to three times its normal size. ${ }^{1}$ Partial enzyme deficiency results in a late-onset phenotype. ${ }^{2}$ Pompe disease has an overall incidence of 1 in 40,000 live births. ${ }^{1,3}$

Electrocardiogram (ECG) abnormalities are universal in Pompe disease because of conduction system abnormalities related to the enlargement of specialized conducting tissue cells with glycogen. ${ }^{4}$ In addition, the topography of the atrioventricular conducting system in Pompe disease is abnormal because of deforming effects of the hypertrophied summit of the ventricular septum. ${ }^{4}$ A shortened PR interval, an increased QT dispersion (QTd), and large LV voltages are hallmarks of this disease. These conduction abnormalities, in conjunction with the hypertrophic cardiomyopathy characteristic of Pompe disease, place these patients at high risk of tachyarrhythmia and sudden death. ${ }^{5-7}$

Although no cure or specific treatment exists for Pompe disease, clinical trials are being performed to evaluate the safety and efficacy of enzyme replacement therapy (ERT). Clinical trials using Chinese hamster ovary $(\mathrm{CHO})$ cell-derived recombinant human GAA (rhGAA) and rhGAA derived from the milk of transgenic rabbits have been studied in the infantile form of Pompe disease. Cardiac response has been dramatic 
with a marked reduction in LV mass index and improved cardiac function..$^{8-11}$ We hypothesized that the conduction abnormalities in infants with Pompe disease would also improve with rhGAA ERT. In this study, we investigated the effect of rhGAA ERT on PR interval, QTc interval, QTd, and LV voltage in infants with Pompe disease.

\section{MATERIALS AND METHODS}

Nineteen ( 4 females/15 males) patients with a median age of 5.5 months (range 2-12 months) were enrolled in open-label clinical trials exploring the safety and efficacy of $\mathrm{CHO}$ cellderived rhGAA ERT at Duke University Medical Center from 1999 to 2004. All patients had a confirmed diagnosis of Pompe disease with an endogenous GAA activity of less than $1 \%$ of the normal mean in skin fibroblasts. GAA activity was assessed by measurement of 4-methyl-umbelliferyl- $\alpha$-D-glucoside cleavage at $\mathrm{pH}$ 4.3. ${ }^{12}$

During this time period, 18 patients received intravenous infusions of $\mathrm{CHO}$ cell-derived rhGAA as part of open-label clinical trials exploring the safety and efficacy of ERT as previously described. ${ }^{8,9}$ One patient died before the start of ERT. The doses ranged from $10 \mathrm{mg} / \mathrm{kg}$ per week to 20 to $40 \mathrm{mg} / \mathrm{kg}$ every 2 weeks. As part of the clinical evaluation, all patients received standard 12-lead ECGs at baseline and repeat ECGs at 4- to 6-week intervals. The studies were approved by the Duke Institutional Review Board, and written informed consent was obtained from each patient's parent/guardian.

\section{Electrocardiograms}

Standard 12-lead ECGs $(10 \mathrm{~mm} / \mathrm{mV}$ and $25 \mathrm{~mm} / \mathrm{sec})$ were obtained using Hewlett-Packard Page Writer Xli recorders (Hewlett-Packard, Palo Alto, CA). ECGs were evaluated manually with calipers by two independent observers who were blinded to the identity of the patient and the treatment status when the ECGs were performed. QT interval and RR intervals were obtained from three consecutive heartbeats. The PR interval was the longest obtainable from any lead. Measurement of the QT was obtained in lead II or lead V5 because these leads are unlikely to have a $\mathrm{U}$ wave, which can obscure the downslope of the $\mathrm{T}$ wave. The end of the $\mathrm{T}$ wave corresponds to restitution of the downslope as it intersects the baseline. In the presence of a $U$ wave, the downslope was extrapolated to the baseline. The QTc interval was obtained using Bazett's formula (QT interval divided by the square root of the preceding R-R interval). QTd was calculated as the difference between the longest and the shortest QT interval. The LV voltage, expressed in millivolts, was determined by the sum of the $\mathrm{S}$ wave in V1 and the $\mathrm{R}$ wave in $\mathrm{V} 6$.

\section{Echocardiograms}

Echocardiographic evaluation consisting of a complete twodimensional cross sectional, M-mode, and Doppler examination was performed using a Siemens Sequoia system (Mountain View, CA) or a Philips 5500 or 7500 system (Bothell, WA) at baseline and at $4,8,12,24,36$, and 52 weeks of rhGAA therapy. LV mass was measured by the area-length method using an average of three to five independent measurements from the same study. ${ }^{13}$

\section{Statistical analysis}

The results from the two independent observers were averaged, and interobserver variability was assessed by regression analysis. Data are expressed as median (range) unless otherwise noted. Group medians using pre-rhGAA ERT ECG and last post-ERT ECG parameters were compared by the Wilcoxon nonparametric rank-sum test. $P$ values of .05 or less were considered statistically significant.

\section{RESULTS}

A total of 134 ECGs from 19 patients were evaluated in this study. All patients had one ECG performed before rhGAA ERT and between 1 and 18 ECGs after the initiation of rhGAA ERT. The interobserver variability correlation coefficients for the various measured parameters were between 0.82 and 0.89 .

The PR and QTc intervals, QTd, and SV1 + RV6 amplitude were plotted over time on therapy and are shown in Figures 1 to 4 . By using the pre-rhGAA ERT ECG versus last post-rhGAA ERT ECG, the various parameters were compared and are demonstrated in Table 1.

The median follow-up period was 6 months (range 2-30 months). The PR interval lengthened from $83(42-110) \mathrm{ms}$ to 107 (95-130) $\mathrm{ms}(P<.001)$, and the QTd decreased from 83 $(40-125) \mathrm{ms}$ to $53(20-80) \mathrm{ms}(P=.003)$. There were significant decreases in LV voltage (67 [17-83] $\mathrm{mV}$ vs. 48 [18-77] $\mathrm{mV}, P=.03)$. There was no evident change in the QTc interval (429 [390-480] ms vs. 413 [370-450] ms, $P=$ not significant). The decrease in LV voltage noted on ECG correlated with a concomitant decrease in LV mass on two-dimensional echo (Fig. 5). A prolonged survival was noted in our patients treated with ERT. Twelve of the patients are currently alive with a mean age of 34.9 months (8.1-69.7 months) and continue to receive ERT. Six patients died at a mean age of 30.6 months (11.8-51.8 months). Thus, the cohort of patients on

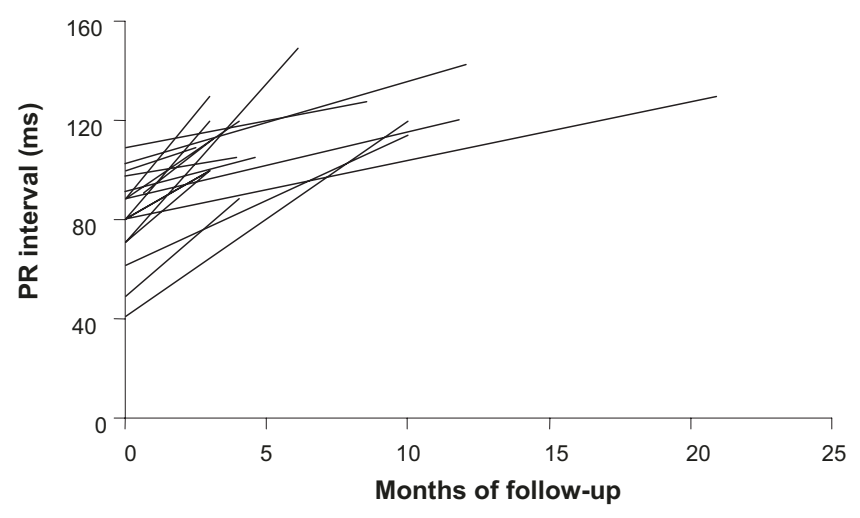

Fig. 1 The individual plots of 18 patients measured in milliseconds before and after enzyme replacement therapy (ERT). The PR interval measured after ERT is from the last electrocardiograph (ECG) on record. 


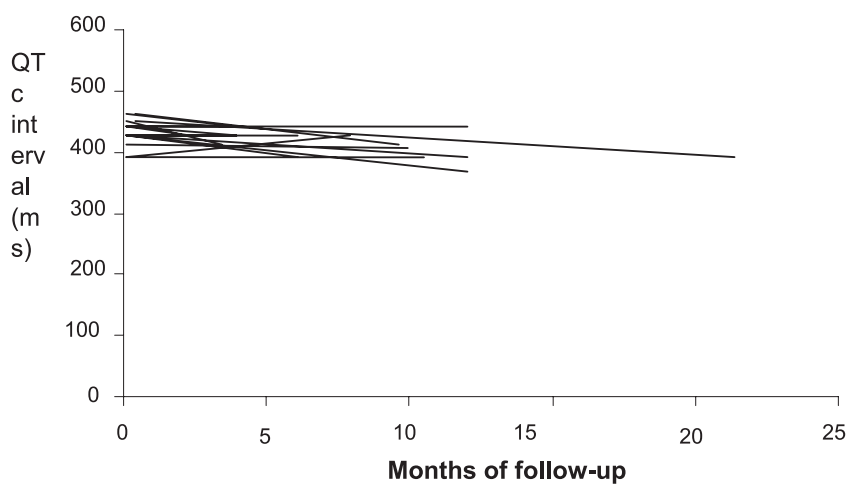

Fig. 2 Plots of QTc interval in milliseconds over months of follow-up.

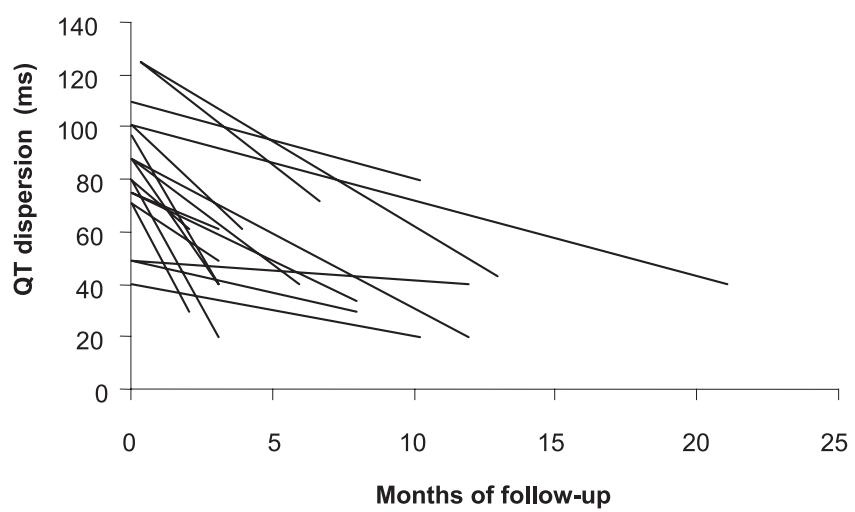

Fig. 3 Plots of QT dispersion in milliseconds over months of follow-up.

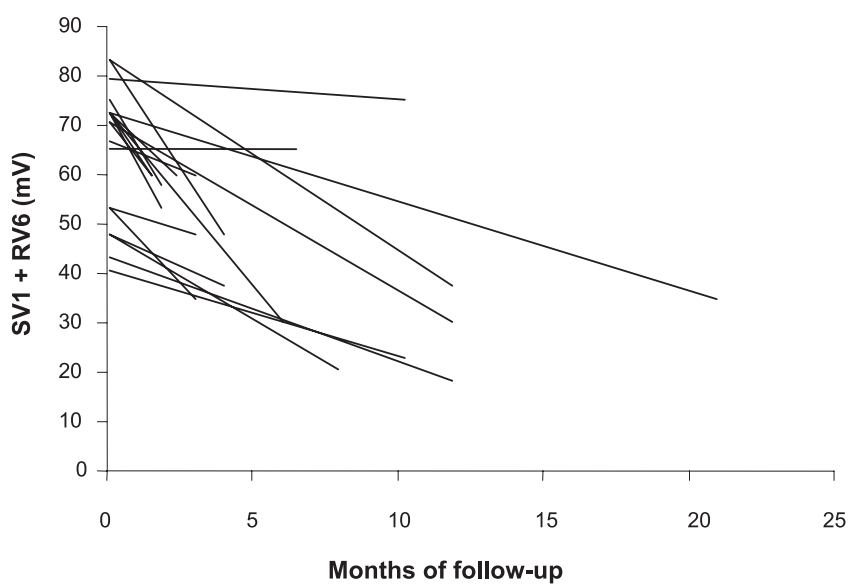

Fig. 4 Plots of left ventricular voltages in millivolts over months of follow-up.

ERT demonstrated markedly extended survival when compared with untreated historical controls. ${ }^{2,14}$

\section{DISCUSSION}

Pompe disease is characterized by a progressive cardiomyopathy and significant conduction abnormalities on ECG. A short PR interval and tall and broad QRS complexes are seen on ECG and often considered diagnostic for Pompe disease. Other storage diseases causing similar pathophysiology in-
Table 1

Pre- and post-median values of different electrocardiographic parameters with concordant $P$ value significance

\begin{tabular}{lcccc}
\hline & $\begin{array}{c}\text { PR } \\
(\mathrm{ms})\end{array}$ & $\begin{array}{c}\text { QTc } \\
(\mathrm{ms})\end{array}$ & $\begin{array}{c}\text { QT disp } \\
(\mathrm{ms})\end{array}$ & $\begin{array}{c}\text { SV1 + RV6 } \\
(\mathrm{mV})\end{array}$ \\
\hline Pre & $83(42-110)$ & $429(390-480)$ & $83(40-125)$ & $67(17-83)$ \\
Post & $107(95-130)$ & $413(370-450)$ & $53(20-80)$ & $48(18-77)$ \\
$P$ value & $<.001$ & NS & .003 & .03 \\
\hline
\end{tabular}

NS, not significant.

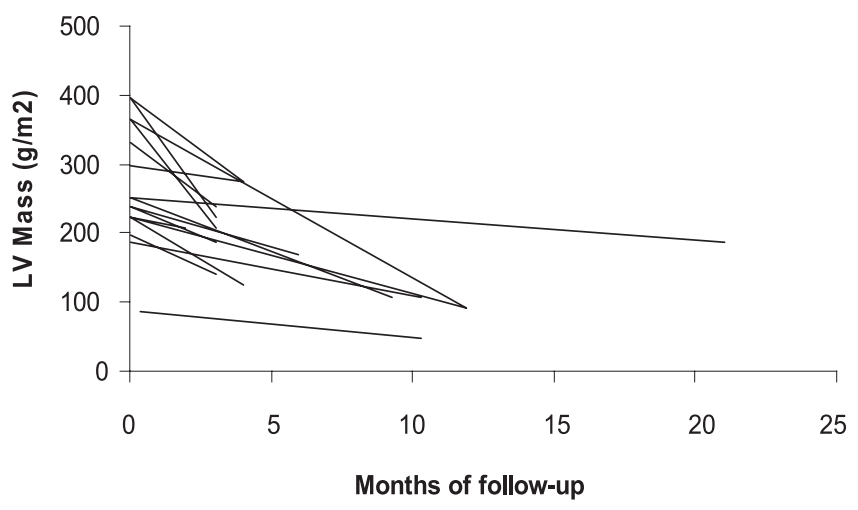

Fig. 5 Plots of left ventricular mass on two-dimensional echo over months of followup.

clude Danon disease caused by mutations of the lysosomeassociated membrane protein 2 gene, resulting in lysosomal glycogen accumulation, and disease caused by mutations in the gene for AMP-activated protein kinase $\gamma_{2}$, both of which result in accumulation of cardiac glycogen leading to left ventricular hypertrophy (LVH). ${ }^{15-17}$ In addition to LVH, both disorders also have associated electrophysiologic abnormalities including a short PR interval. ${ }^{15}$ Prior studies of Pompe disease in mouse models have not dealt extensively with electrophysiologic cardiac abnormalities. These studies in Pompe mice are warranted; experience from the mouse models for lysosomeassociated membrane protein 2 and AMP-activated protein kinase $\gamma_{2}$ mutations can help us to better understand cardiac arrhythmias in lysosomal storage diseases like Pompe. . $^{15,16,18,19}$

With ERT, we and others have demonstrated an improvement in the hypertrophic cardiomyopathy in Pompe disease by two-dimensional echocardiography. ${ }^{8-11}$ This study is the first to systematically examine the ECG changes in a cohort of patients with Pompe disease in response to ERT with rhGAA. We report changes in ECG findings including an increase in the PR interval, decrease in LV voltage, and shortening of QTd after initiation of ERT.

Baseline characteristic ECG findings in this study showed a shortened PR interval, LVH, and increased QTd. Similar baseline ECG findings in patients with infantile-onset Pompe disease were reported in a large retrospective chart review of 150 patients. In this review, ECG data were available from $137 \mathrm{pa}-$ tients (81.5\%). Evidence of LVH was present in all but two patients. Short PR intervals were reported in up to $56.3 \%$ of the patients. ${ }^{14}$ 
The normal PR interval is $110 \mathrm{~ms}(80-130 \mathrm{~ms}$ [5th-95th percentiles]) for infants aged 1 to 6 months and $120 \mathrm{~ms}$ (100$140 \mathrm{~ms}$ [5th-95th percentiles]) for children aged 6 months to 3 years. ${ }^{20}$ When we applied these norms in our population and adjusted for gestational age, approximately $75 \%$ (14/19) of cases had PR intervals at the fifth percentile or less at baseline. Of the five remaining patients, two had PR intervals that were between the 10th and 25th percentiles, and the remaining three patients had PR intervals between the 25th and 50th percentiles.

All patients in our study had a progressive increase in the PR interval with rhGAA therapy. Similarly, in Fabry disease, which is associated with cardiac involvement including a short PR interval, ERT resulted in an increase in PR interval to normal after 6 months of ERT. ${ }^{21,22}$ In 1982, Bharati et al. studied the conduction system in patients with Pompe disease correlating pathologic and electrophysiologic findings. ${ }^{4}$ They concluded that the short PR interval on the ECG may be related to the enlargement of cells, which may in turn be related to increased glycogen content because the rapid conduction was not localized in the anatomic counterpart of the HV interval. The relationship of glycogen per se to the speed of conduction is unknown. ${ }^{4}$ Functional changes in cell-to-cell connections, depolarizing channel density, channel functionality, and local autonomic innervation will also alter net tissue conduction velocity and are unexplored in this disease.

LVH causes the characteristic high-voltage QRS complexes seen on ECG. ${ }^{23}$ We observed that LVH, based on voltages in V1 and V6, decreases with ERT. LVH is caused by the glycogen accumulation in the cardiac muscle, which leads to thickening of the walls of both ventricles and interventricular-septum, resulting in a hypertrophic cardiomyopathy. ${ }^{1}$ In particular, the thickness of the LV posterior wall increases significantly over time. ${ }^{2}$ Histologic studies performed on patients with Pompe disease and knockout mice models of Pompe disease have demonstrated characteristic periodic acid-Schiff positive and acid phosphatase positive stained vacuoles, identified as lysosomes filled with glycogen. ${ }^{18}$ The decrease in LVH that we observed on ECG correlates with two-dimensional ECG findings (Table 2) and previously reported improvement in the hypertrophic cardiomyopathy by two-dimensional echocardiography with ERT. ${ }^{8-11}$

We also demonstrated significant changes in QTd, which is an indirect measure of the heterogeneity of ventricular repo-

\section{Table 2}

Pre- and post-median values comparing left ventricular voltages

(electrocardiography) with left ventricular mass (two-dimensional echocardiography) in 18 patients who received enzyme replacement therapy

\begin{tabular}{lcc}
\hline & $\begin{array}{c}\text { SV1 }+\mathrm{RV} 6 \\
(\mathrm{mV})\end{array}$ & $\begin{array}{c}\text { LV mass } \\
\left(\mathrm{g} / \mathrm{m}^{2}\right)\end{array}$ \\
\hline Pre & $67(17-83)$ & $249(64-404)$ \\
Post & $48(18-77)$ & $129(42-273)$ \\
$P$ value & .03 & $<.00003$ \\
\hline
\end{tabular}

$\mathrm{LV}$, left ventricular. larization after ERT. Normal values of QTd are reported to be $36 \pm 13.7 \mathrm{~ms}$ in 111 healthy children 5 days to 16 years old..$^{24}$ There is increased QTd in various cardiac diseases including patients with LVH and hypertrophic cardiomyopathy. ${ }^{25,26 \mathrm{Al}-}$ though the mechanism by which QTd is increased in hypertrophic cardiomyopathy remains unclear, increased QTd has been shown to be a marker of increased risk for sudden cardiac death. ${ }^{27}$ The myocardial disarray and fibrosis characteristic of hypertrophic cardiomyopathy have been implicated in causing the spatial dispersion of the cardiac impulse supporting reentrant arrhythmias, although the exact mechanisms are unknown. ${ }^{25,27}$ Electrophysiologic studies demonstrate that the inducibility of ventricular tachycardia in patients with hypertrophic cardiomyopathy correlates with increased differences in refractoriness between the right ventricular outflow tract and the right ventricular apex..$^{25,28}$

Because progressive accumulation of glycogen in cardiac tissue is the most probable cause of the conduction abnormalities seen in Pompe disease, we postulate that ERT reduces glycogen deposition in the myocardium and conduction system, thereby increasing the PR interval and decreasing the LV voltage. How reduction of the glycogen in the heart decreases QTd is uncertain. The effects of cell size, cell-to-cell coupling, interstitial fibrosis, intramyocyte conductivity, and the autonomic nervous system may all influence repolarizing currents.

Our study is limited by our results reflecting pooled data over different periods using varying doses of ERT. Pooling potentially heterogeneous data to provide a uniform estimate of effect may not be appropriate; however, we did not attempt to provide a uniform estimation of dose effect. For this reason, we have depicted the raw data in the figures. Nevertheless, all patients followed for more than 2 months seem to respond dramatically with progressive changes in the PR interval, QTd, and LV voltage. Furthermore, a universal cardiac response was noted in all 18 of the patients treated with rhGAA ERT, irrespective of disease severity at treatment outset. All patients had a decrease in LV mass and an improvement in cardiac function on two-dimensional echo. The improvement in LVH correlated with improvements on ECG, particularly LVH (Table 2).

In conclusion, serial ECGs can serve as a useful adjunct to two-dimensional echocardiography in following the cardiac response of patients with infantile Pompe disease receiving ERT.

Our results are promising and continue to add to the growing evidence of beneficial effects of ERT in treating Pompe disease. ${ }^{29}$ Future efforts should evaluate the effect of long-term use of ERT in cardiac remodeling in patients with Pompe disease in multicenter clinical trials to determine whether the long-term risk of tachyarrhythmia and sudden death changes with this novel therapy.

\section{ACKNOWLEDGMENTS}

We thank Joanne Mackey, PNP, and Stephanie DeArmey, PA, for the excellent clinical care of these critically ill babies. The research supported in this article was supported by a grant from Genzyme Corporation and by M01-RR30, National Cen- 
ter for Research Sources, General Clinical Research Centers Program. P.S.K. and Y.T.C. received research/grant support from Genzyme Corporation; P.S.K. is a member of the Pompe Disease Registry Advisory Board for Genzyme Corporation. Y.T.C has served as a consultant for Genzyme Corporation. If therapy for Pompe disease proves successful commercially, Duke University and inventors for the cell line used to generate the enzyme (rhGAA) used in this clinical trial may benefit financially pursuant to the University's Policy on Inventions, Patents, and Technology Transfer.

\section{References}

1. Kishnani PS, Howell RR. Pompe disease in infants and children. J Pediatr 2004;144: S35-S43.

2. van den Hout HM, Hop W, van Diggelen OP, Smeitink JA, et al. The natural course of infantile Pompe's disease: 20 original cases compared with 133 cases from the literature. Pediatrics 2003;112:332-340.

3. Hirschhorn R, Reuser AJ. Glycogen storage disease type II: acid alpha-glucosidase (acid maltase) deficiency. In: The Metabolic and Molecular Bases of Inherited Disease. Scriver C, Beaudet A, Sly W, Valle D, et al (Eds.). New York: McGraw Hill, 2001: 3389-3420.

4. Bharati S, Serratto M, DuBrow I, Paul MH, et al. The conduction system in Pompe's disease. Pediatr Cardiol 1982;2:25-32.

5. Bulkley BH, Hutchins GM. Pompe's disease presenting as hypertrophic myocardiopathy with Wolff-Parkinson-White syndrome. Am Heart J 1978;96:246-252.

6. Francesconi M, Auff E, Ursin C, Sluga E. [WPW syndrome combined with AV block 2 in an adult with glycogenosis (Type II)]. Wien Klin Wochenschr 1982;94:401-404.

7. Tabarki B, Mahdhaoui A, Yacoub M, Selmi H, et al. [Familial hypertrophic cardiomyopathy associated with Wolff-Parkinson-White syndrome revealing type II glycogenosis]. Arch Pediatr 2002;9:697-700.

8. Amalfitano A, Bengur AR, Morse RP, Majure JM, et al. Recombinant human acid alpha-glucosidase enzyme therapy for infantile glycogen storage disease type II results of a phase I/II clinical trial. Genet Med 2001;3:132-138.

9. Kishnani P, Voit T, Nicolino M, Tsai C, et al. Enzyme replacement therapy with recombinant human acid alpha glucosidase (rhGAA) in infantile Pompe disease (IPD): results from a phase 2 study. Pediatr Res 2003;53:259A.

10. Klinge L, Straub V, Neudorf U, Schaper J, et al. Safety and efficacy of recombinant acid alpha-glucosidase (rhGAA) in patients with classical infantile Pompe disease: results of a phase II clinical trial. Neuromuscul Disord 2005;15:24-31.

11. Van den Hout H, Reuser AJ, Vulto AG, Loonen MC, et al. Recombinant human alpha-glucosidase from rabbit milk in Pompe patients. Lancet 2000;356:397-398.
12. Van der Ploeg AT, Hoefsloot LH, Hoogeveen-Westerveld M, Petersen EM, et al. Glycogenosis type II: protein and DNA analysis in five South African families from various ethnic origins. Am J Hum Genet 1989;44:787-793.

13. Reichek N, Helak J, Plappert T, Sutton MS, et al. Anatomic validation of left ventricular mass estimates from clinical two-dimensional echocardiography: initial results. Circulation 1983;67:348-352.

14. Corzo D, Hwu WL, Mandel H, Nicolino M, et al. Natural history of infantile-onset Pompe disease: results from a retrospective chart review study. Mol Genet Metab 2004;82:170.

15. Arad M, Maron BJ, Gorham JM, Johnson WH Jr, et al. Glycogen storage diseases presenting as hypertrophic cardiomyopathy. N Engl J Med 2005;352:362-372.

16. Arad M, Moskowitz IP, Patel VV, Ahmad F, et al. Transgenic mice overexpressing mutant PRKAG2 define the cause of Wolff-Parkinson-White syndrome in glycogen storage cardiomyopathy. Circulation 2003;107:2850-2856.

17. Charron P, Villard E, Sebillon P, Laforet P, et al. Danon's disease as a cause of hypertrophic cardiomyopathy: a systematic survey. Heart 2004;90:842-846.

18. Bijvoet AG, van de Kamp EH, Kroos MA, Ding JH, et al. Generalized glycogen storage and cardiomegaly in a knockout mouse model of Pompe disease. Hum Mol Genet 1998;7:53-62.

19. Raben N, Nagaraju K, Lee E, Kessler P, et al. Targeted disruption of the acid alphaglucosidase gene in mice causes an illness with critical features of both infantile and adult human glycogen storage disease type II. J Biol Chem 1998;273:19086-19092.

20. Park MK. Pediatric Cardiology for Practitioners, 3rd ed. St. Louis: Mosby, 1996

21. Goldman ME, Cantor R, Schwartz MF, Baker M, et al. Echocardiographic abnormalities and disease severity in Fabry's disease. J Am Coll Cardiol 1986;7:1157-1161.

22. Waldek S. PR interval and the response to enzyme-replacement therapy for Fabry's disease. N Engl J Med 2003;348:1186-1187.

23. Jacob JL, Leandro RL, Parro Junior A. Pompe's disease or type IIa glycogenosis. Ar Qbras Cardiol 1999;73:435-440.

24. Tutar HE, Ocal B, Imamoglu A, Atalay S. Dispersion of QT and QTc interval in healthy children, and effects of sinus arrhythmia on QT dispersion. Heart 1998;80: 77-79.

25. Dritsas A, Sbarouni E, Gilligan D, Nihoyannopoulos P, et al. QT-interval abnormalities in hypertrophic cardiomyopathy. Clin Cardiol 1992;15:739-742.

26. Malik M, Batchvarov VN. Measurement, interpretation and clinical potential of QT dispersion. J Am Coll Cardiol 2000;36:1749-1766.

27. Yi G, Elliott P, McKenna WJ, Prasad K, et al. QT dispersion and risk factors for sudden cardiac death in patients with hypertrophic cardiomyopathy. Am J Cardiol 1998;82:1514-1519.

28. Watson RM, Schwartz JL, Maron BJ, Tucker E, et al. Inducible polymorphic ventricular tachycardia and ventricular fibrillation in a subgroup of patients with hypertrophic cardiomyopathy at high risk for sudden death. J Am Coll Cardiol 1987; 10:761-774

29. Chen YT, Amalfitano A. Towards a molecular therapy for glycogen storage disease type II (Pompe disease). Mol Med Today 2000;6:245-251. 